\title{
Muslimische Helden: \\ Die Kreuzzüge im Musiktheater
}

\author{
Albert Gier
}

Das Thema der Kreuzzüge gerät im frühneuzeitlichen Europa vor allem über die Rezeption der beiden großen Ritterromanzi aus Ferrara, von Ludovico Ariostos Orlando furioso (1516/1532) und Torquato Tassos Gerusalemme liberata (1581), ins Blickfeld - wobei die Geschichte vom Spanienfeldzug Karls des Großen, die dem Orlando furioso zugrunde liegt, die Thematik in zeitliche Ferne rückt und sagenhaft überhöht: Um 1100, also zur Zeit des Ersten Kreuzzugs, entstand die älteste, in der Handschrift der Bodleiana in Oxford überlieferte Fassung des altfranzösischen Rolandlieds. ${ }^{1}$ In der Auseinandersetzung zwischen christlichen Franken und ,Heiden', das heißt Muslimen, ergreift der anonyme Autor (wie kaum anders zu erwarten) dezidiert und einseitig Partei für die christliche Seite: „Païen unt tort e chrestien unt dreit"

Von dieser holzschnittartigen Sichtweise mag Torquato Tasso nicht allzu weit entfernt gewesen sein: Seine Gerusalemme liberata ist durchaus als Kreuzzugspropaganda, als Appell an die Zeitgenossen zu verstehen, es Gottfried von Bouillon und seinen Mitstreitern gleichzutun (zur Zeit der osmanischen Expansion im östlichen Mittelmeerraum unter Soliman II. [1520-1566] hatte das Thema neue Aktualität gewonnen). Ariost dagegen hat die versunkene Welt der Ritter aus ironischer Distanz behandelt. Die kriegerische Auseinandersetzung zwischen Christentum und Islam, Orient und Okzident (konkret: die Belagerung von Paris durch den nordafrikanischen Herrscher Agramante) bildet im Orlando furioso lediglich den Hintergrund einer bunten Reihe von Episoden um prominente (christliche wie muslimische) Kämpfer, die sich persönlichen Ruhm, und vor allem die Liebe einer Dame zu erwerben suchen. ${ }^{3}$ Dabei richtet sich das Begehren der meisten Ritter auf Angelica, die wunderschöne Tochter des Königs von Catai (in China), die Matteo Maria Boiardo als Störfaktor und Unruhestifterin in seinen Orlando innamorato (1486) eingeführt hat, an den Ariost anschließt.

1 La chanson de Roland, hg. von Cesare Segre (Dokumenti di filologia; 16). Mailand [u.a.] 1971.

2 Ebd., V. 1015.

3 Vgl. zuletzt Paul Geyer: Von Dante zu Ionesco. Literarische Geschichte des modernen Menschen, Bd 1: Dante, Petrarca, Boccaccio, Machiavelli, Ariost, Tasso. Hildesheim [u.a.] 2013, 212-222, hier 215. 
Gleich im ersten Canto des Orlando furioso ${ }^{4}$ findet sich eine emblematische Szene: ${ }^{5}$ Angelica, die Karl der Große in die Obhut eines älteren Herrn gegeben hat, damit sie seine Ritter nicht länger durcheinanderbringt, flieht zu Pferd und wird von Rinaldo verfolgt - zu Fuß, denn in einer Schlacht, welche die Christen verloren haben, ist ihm sein Pferd Baiart abhandengekommen. Der Sarazene Ferraù versucht unterdessen vergeblich, seinen Helm, der ihm in den Fluß gefallen ist, herauszuangeln; auf Angelicas Hilferufe hin attackiert er ihren Verfolger. Wie Rinaldo ist auch Ferraù verliebt in Angelica, die sich davonmacht, ohne das Ende des Kampfes abzuwarten. Der Franke gibt daraufhin seinem Gegner zu bedenken, dass unter diesen Umständen keiner von ihnen eine Chance hat, die Dame für sich zu gewinnen; es wäre besser, einen Waffenstillstand zu schließen, Angelica einzufangen und dann erst um sie zu kämpfen (ob das Objekt ihrer Begierde den einen, den anderen oder einen Dritten bevorzugt, scheint belanglos). Ferraù ist einverstanden, er lässt den fränkischen Fußgänger sogar mit auf seinem Pferd reiten. Der Erzähler kommentiert:

Oh gran bontà de' cavallieri antiqui!

Eran rivali, eran di fé diversi,

E si sentian degli aspri colpi iniqui

per tutta la persona anco dolersi;

e pur per selve oscure e calli obliqui

insieme van senza sospetto aversi. ${ }^{6}$ (OF I, 22, V. 1-6)

Christliche wie muslimische Ritter sind bei Ariost Repräsentanten einer kulturübergreifenden Elite, die sich durch gemeinsame Wertvorstellungen und ein ähnliches Lebensgefühl miteinander verbunden weiß. Die Bewunderung des Erzählers wird freilich mehrfach ironisch gebrochen: Rinaldo, der Ritter ohne Ross, und der nach seinem Helm fischende Ferraù geben beide ein leicht groteskes Bild ab, das nicht unbedingt ihre Vortrefflichkeit (bontà) spiegelt. Auch die zwei Männer auf einem Pferd wirken ein bisschen lächerlich; und der Leser erinnert sich, dass Rinaldo (,il buon figliol d'Amone“ [OF I, 21, V. 6]) zu den Söhnen Haimons gehört, die alle vier bequem Platz auf dem Rücken Baiarts hatten. ${ }^{7}$ Da der leider durchgegan-

4 Ludovico Ariosto: Orlando furioso [=OF], hg. von Cesare Segre (I Meridiani collezione). Mailand 2006.

5 OF I, 10-26.

6 „O Biederkeit der alten Rittersitten! | Die Nebenbuhler waren, die entzweit | Im Glauben waren, bittern Schmerz noch litten | Am ganzen Leib vom feindlich wilden Streit, | Frei von Verdacht und in Gemeinschaft ritten | Sie durch des krummen Pfades Dunkelheit." (Ariost: Der rasende Roland, 2 Bde., übers. von Johann Dietrich Gries (1804-1809). München 1980, Bd. 1, 10-11. It. bontà ist mit ,Biederkeit' kaum angemessen wiedergegeben.)

7 Rinaldo ist der Renaut de Montauban der altfranzösischen Chanson de geste (Renaut de Montauban oder Les Quatre Fils Aymon, Ende 12. Jahrhundert); in Italien war der Stoff spätestens seit dem 15. Jahrhundert bekannt (Innamoramento di Rinaldo da Monte-Albano, gedruckt 1474). Nachdem der junge Ritter Renaut-Rinaldo von Karl dem Großen zum Tode verurteilt worden ist, weil er im Streit seinen Neffen erschlagen hat, flieht er mit seinen Brüdern auf Baiart in die Ardennen; vgl. Werner Wunderlich: Haimonskinder. In: Rolf 
gen ist, ${ }^{8}$ muss sich Rinaldo für einmal mit einem weniger prominenten Reittier begnügen. Und schließlich ist die Überlegung, die der Paladin anstellt - man muss den Bären (oder die Dame) erst erlegen, bevor man das Fell verteilen kann - kaum als besonders chevalersk zu bezeichnen.

Im Orlando furioso werden christlich-muslimische Liebesgeschichten als zumindest möglich dargestellt. So verliebt Rodomonte sich in Isabella und Angelica hat Dutzende fränkischer Verehrer. Im Zentrum stehen die Heldenjungfrau Bradamante, Rinaldos Schwester, und der Sarazenenkrieger Ruggiero, in denen Ariost wie Boiardo die Stammeltern des Hauses Este sieht, in dessen Auftrag der Orlando innamorato wie der Furioso entstanden. Freilich waren Ruggieros Vorfahren Christen, ${ }^{9}$ und er selbst fühlt sich innerlich längst der Religion seiner Ahnen zugehörig ${ }^{10}$ und wäre nicht nur um Bradamantes willen ohne Weiteres bereit zu konvertieren. Er zögert nur deshalb, weil sich das Kriegsglück unterdessen gegen Agramante gewendet hat: Seinen Herrn zu diesem Zeitpunkt im Stich zu lassen, wäre unedel. ${ }^{11}$ Was das Verhältnis der Geschlechter betrifft, scheint es zwischen Christen und Nichtchristen kaum kulturelle Unterschiede zu geben: Angelica ${ }^{12}$ manipuliert ihre fränkischen und sarazenischen Verehrer mit gleicher Virtuosität. Dass sie sich zuletzt in einen Muslim verliebt, scheint Zufall, es hätte ebensogut ein Christ sein können.

Das Frauenbild christlicher wie muslimischer Ritter scheint bei Ariost von einer merkwürdigen Ambivalenz geprägt: Einerseits ist die Dame die Gebieterin, deren Gunst sich der Mann durch Gehorsam und nie ermüdenden Einsatzwillen verdienen muss, andererseits wird sie als Beute betrachtet und, wenn sich die Gelegenheit bietet, auch behandelt ${ }^{13}$ - genau das haben zum Beispiel Rinaldo und Ferraù im ersten Canto mit Angelica vor. Dass die Christen als potenzielle Vergewaltiger erscheinen, mochten Leser und Opernbesucher des 17. und 18. Jahrhunderts weniger erstaunlich finden als die Bereitschaft der Sarazenen, um Angelicas oder anderer Damen willen mühevolle und langwierige Abenteuer auf sich zu nehmen, denn die Vorstellung, die sich europäische Männer vom Verhältnis der Geschlechter in der islamischen Welt machen, scheint in jener Zeit maßgeblich von der so fremdartigen Einrichtung des Harems bestimmt: Reiche und mächtige Orientalen, so

Wilhelm Brednich (Hg.): Enzyklopädie des Märchens. Handwörterbuch zur historischen und vergleichenden Erzäblforschung, Bd 6. Berlin und New York 1990, 385-396.

8 OF I, 12, V. 3.

9 Vgl. OF XXII, 35; XXXVI, 70-72 (Hector von Troja als Stammvater seiner Familie).

10 OF XXII, 35, V. 1/2; XXV, 89, V. 5/6.

11 Vgl. seinen Brief an Bradamante: OF XXV, 86-89.

12 Da sie aus Catai, also aus China stammt, dürfte sie weder Christin noch Muslimin sein. Ihre Religionszugehörigkeit wird weder bei Ariost noch bei Boiardo thematisiert; in der Auseinandersetzung zwischen Franken und Sarazenen steht sie auf der Seite dieser letzten (Boiardo zufolge wollten Angelica und ihr Bruder möglichst viele von Karls Rittern in eine Falle locken und gefangen nehmen, um die Kampfkraft der Franken zu schwächen).

13 Dazu Albert Gier: Ludovido Ariostos Orlando furioso: die Dichtung des Sowohl - Als auch. In: Italienische Studien 7, 1984, 5-21, hier 15-21. 
denkt es sich etwa Montesquieu in den Lettres persanes ${ }^{14}$ (1721), kaufen sich Frauen, die als willenlose Objekte einzig und allein dem Vergnügen ihres Besitzers zu dienen haben. Für das Publikum Vivaldis oder Händels war vermutlich ausgemacht, dass auch Ferraù, Sacripante oder Rodomonte einen Harem zu Hause hatten. Wenn sie dennoch ohne viel Aussicht auf Erfolg Angelica hinterherlaufen, statt sich daheim verwöhnen zu lassen, zeigt das, dass sie vorrangig dem durch die Romanliteratur vermittelten, konfessionsübergreifenden Klischee des fahrenden Ritters und erst in zweiter Linie zeittypischen Vorstellungen vom Lebensstil der orientalischen (muslimischen) Oberschicht entsprechen.

Allein die Opernadaptationen von Episoden des Orlando furioso bieten eine Fülle von für unsere Fragestellung relevanten Aspekten, die im Rahmen eines solchen Beitrags unmöglich vollständig behandelt werden können. Im Folgenden weise ich schlaglichtartig auf wenige repräsentative Tendenzen in den AriostOpern hin, gehe dann kontrastiv auf ein Beispiel für Tasso-Rezeption in der Opera seria ein und skizziere abschließend die Sicht der romantischen Oper auf die Kreuzzugsthematik.

Giulio Rospigliosi setzt im Libretto Il palazzo incantato ${ }^{15}$, das er für Luigi Rossi schrieb (Rom 1642), Atlantes Zauberschloss in Szene, das mit Recht als mise en abyme von Ariosts Romanzo bezeichnet wurde: ${ }^{16}$ Der Magier Atlante will verhindern, dass Ruggiero und Bradamante zusammenkommen, denn gemäß einer Prophezeiung soll Ruggiero bald nach ihrer Heirat den Tod finden. Er erschafft deshalb einen Palast, in dem Ritter durch ein Trugbild und eine Stimme festgehalten werden, in denen jeder die Geliebte zu erkennen glaubt; ${ }^{17}$ Rospigliosi versammelt dort die Protagonisten bekannterer und weniger bekannter Episoden aus dem Orlando furioso, die über die Liebe und ihre Sehnsucht nach der geliebten Person räsonieren. Ruggiero und Bradamante sind die Protagonisten des einzigen durchgehenden Handlungsstrangs (Bradamante wird eifersüchtig, wenn sie Rugggiero mit Angelica sieht, erst im Schlussakt versöhnen sich die beiden), vielen anderen Figuren geben kurze Auftritte die Gelegenheit, ihre jeweilige Geschichte zu rekapitulieren. Sie alle scheinen die gleichen Wünsche und Nöte zu haben, unabhängig von ihrer Religionszugehörigkeit: Am Ende des I. Akts finden sich Christen (Bra-

14 [Charles-Louis Baron de] Montesquieu: Lettres persanes (Classiques Garnier), hg. von Paul Verrière. Paris 1971; vgl. die Briefe Nr. 2, Nr. 20 und vor allem die Brieffolge Nr. 147-161 zur Bestrafung der angeblich untreuen Frauen.

15 Text abrufbar unter http://www.librettidopera.it/palinc/palinc.html, 29. März 2016 [=PI]. Vgl. Volker Kapp: Das Barberini-Theater und die Bedeutung der römischen Kultur unter Urban VIII. Versuch einer literaturhistorischen Einordnung des Schaffens von Giulio Rospigliosi. In: Literaturwissenschaftliches Jahrbuch, Neue Folge 26, 1985, 75-100.

16 Vgl. Brigitte Burrichter: Der Zauberer und der Autor - Atlantes Zauberwelt als mise en abyme des romanzo. In: Cornelia Klettke und Georg Maag (Hg.): Trugbildnerisches Labyrinth - Kaleidoskopartige Effekte. Neurezeptionen des Orlando furioso von Ariosto (Horizonte; 9). Tübingen 2006, 17-26.

17 Vgl. OF XII, 1-22. 
damante, Orlando, Fiordiligi) und Muslime (Ferraù, Mandricardo, Marfisa) zusammen in der Klage: „Oh, quanto è duro il non trovar, chi s'ama!“ (PI I 15, S. 31) Im II. Akt sagt Atlante Angelica die Begegnung mit Medoro voraus, in den sie sich verlieben wird. ${ }^{18} \mathrm{Ob}$ der junge Mann Christ oder Muslim ist, erwähnt er nicht, und sie fragt auch nicht danach. Rospigliosis Personal bildet eine interreligiöse und interkulturelle Gemeinschaft von Liebenden - die kleine Minderheit der Verächter der Liebe besteht übrigens aus der Muslimin Marfisa ${ }^{19}$ und dem christlichen Ritter Astolfo ${ }^{20}$, das heißt, selbst hier herrscht Parität!

Von Atlantes Zauberschloss und von der Verbindung Bradamantes mit Ruggiero ist auch in Ortensio Mauros Drama Orlando Generoso für Agostino Steffani ${ }^{21}$ (Hannover 1692) die Rede, im Zentrum stehen hier allerdings Angelica, Orlando und Medoro. Mauro - dadurch, so scheint es, unterscheidet sich sein Buch von allen anderen Opern nach Ariost - verlagert die Handlung nach Catai, in Angelicas Heimat: Die Prinzessin ist mit ihrem geliebten Medoro heimgekehrt, aber auch Orlando, Ruggiero und Bradamante haben den Weg in den Fernen Osten gefunden.

Ariost hat darauf verzichtet, Medoro mit Orlando zu konfrontieren: Nachdem Angelica den verwundeten Medoro gesund gepflegt hat, macht sich das glückliche Paar auf die Heimreise. Orlando findet ihr Liebesnest, erkennt, dass Angelica für ihn verloren ist, und verliert prompt den Verstand. Bei einer letzten, flüchtigen Begegnung erkennt er sie nicht mehr. ${ }^{22}$ Der Leser erfährt auch nicht, wie sich die Beziehung zwischen Angelica und Medoro entwickelt: Die Prinzessin schließt natürlich eine Mesalliance, denn Ariosts Medoro ist zwar mutig und tapfer, ${ }^{23}$ aber von niederer Herkunft. ${ }^{24}$ Vermutlich wird sie befehlen, und er wird gehorchen, aber der Dichter sagt es uns nicht.

Ortensio Mauros Medoro dagegen unterwirft sich Angelica vollständig; ${ }^{25}$ wenn die beiden in eine prekäre Lage geraten - nicht nur Orlando erhebt Anspruch auf Angelica, auch ihr eigener Vater verliebt sich in seine Tochter, die er nicht wieder-

18 PI II 10.

19 PII 3.

20 PI II 13.

21 Ortensio Mauro: Orlando Generoso [=OG]. Drama per il Theatro d'Hannover MDCXCII [1692], http://diglib.hab.de/drucke/textb-320/start.htm, 29. März 2016. Vgl. auch Albert Gier: Damen, Ritter, Waffen und Liebe. Ariosts Orlando furioso auf der Opernbühne. In: Händel-Jabrbuch 57, 2011, 307-332, hier 327.

22 OF XXIX, 58-66: Den wahnsinnigen Orlando hat es nach Spanien verschlagen; an der Küste trifft er das Liebespaar, das im Begriff ist, sich nach China einzuschiffen. Obwohl er Angelica nicht erkennt, fühlt er Begehren und verfolgt sie; sie benutzt ihren Zauberring, um sich unsichtbar zu machen, und fällt vom Pferd, dessen sich Orlando bemächtigt. In den folgenden Canti ist von Angelica nicht mehr die Rede.

23 Er wagt (aus eigenem Antrieb) sein Leben, um seinen toten König angemessen zu begraben (OF XVIII, 167-XIX, 13), und wird dabei schwer verwundet.

24 OF XVIII, 165, V. 2.

25 Vgl. OG II 13, S. 76: „Ciò ch’à te piace, approvo, | Mi son legge sourana i tuoi uoleri.“ 
erkennt $^{26}$-, bleibt Medoro ganz passiv und überlässt es der Geliebten, einen Ausweg zu finden. Im Gegensatz zu allen anderen Figuren im Libretto ${ }^{27}$ ist er nicht eifersüchtig, obwohl er am meisten Grund dazu hätte, und vertraut Angelica blind. 28

Einerseits ist die Macht der Liebe universell, niemand, weder Christ noch Muslim, kann ihr widerstehen, so stellt es Atlante fest. ${ }^{29}$ Andererseits gibt es sehr wohl Gradunterschiede: Angelica gesteht ein, dass sie „per un bel sembiante, il fasto mio, | La gloria, la fortuna, | La patria, e l' Genitor posi in oblio" (OG III 10, S. 109), womit sich die Thronerbin ${ }^{30}$ von Catai extrem verantwortungslos verhält. Orlando weiß, dass er in König Galafros Reich nichts zu suchen hat, eigentlich soll er im belagerten Paris an Karls Seite kämpfen, ${ }^{31}$ aber die Liebe zu Angelica ist (noch) stärker als sein Pflichtgefühl. Zuletzt freilich wird Orlando seinen Egoismus überwinden, auf Angelica verzichten und seine Aufgabe als miles christianus erfüllen.

Im Orlando furioso, und auch in Ortensio Mauros Libretto, ${ }^{32}$ ist Medoros Hilfsbedürftigkeit, die bei Angelica zuerst Mitleid, dann Liebe hervorruft, ${ }^{33}$ eine Folge seiner Tapferkeit: Er hat gegen eine Übermacht gekämpft und ist verwundet worden. Andere Librettisten machen ihn zum Schwächling: In Grazio Bracciolis Orlando (1727, Musik Antonio Vivaldi ${ }^{34}$ ) vermag er sich im Kampf mit Ruggiero nicht zu behaupten, denn, so muss er sich von Angelica sagen lassen, seine Fähig-

26 Zweifel an seiner Aufrichtigkeit scheinen angebracht: Orlando weist ihn ausdrücklich darauf hin, dass er seine Tochter vor sich hat, aber Galafro glaubt ihm nicht (OG II 6); seine Aussage „Di così bel sembiante | Godo in cambio di Padre esser Amante“ (OG II 6, S. 62) klingt irritierend zweideutig.

27 Bradamante wird eifersüchtig, wenn sie Ruggiero mit Angelica sieht, die er vor Räubern gerettet hat (OG I 14), und macht ihm eine Szene (OG II 4); Orlando beobachtet Angelica und Medoro, die Eifersucht läßt ihn den Verstand verlieren (OG II 10); der Wahnsinnige hält Bradamante für Angelica und spricht zärtlich mit ihr (OG II 11), was Ruggiero, der von Orlandos Zustand noch nichts weiß, eifersüchtig macht (OG II 12); Galafro wird eifersüchtig, wenn er hört, Angelica sei „mit einem Krieger“ (Medoro) gesehen worden (OG III 5).

28 Vgl. OG II 9: „Pur che tu sia leale | Non mi curo di Rè ne di Riuale.“

29 OG II 20: „Così senza piazer uan tutti errando, | E tien ogn'un de la pazzia d'Orlando.“ Aus dem Zusammenhang wird klar, dass das Zauberschloss allegorisch als das erotische Begehren zu verstehen ist, dem niemand zu widerstehen vermag.

30 Ihr Bruder Argalia ist von Ferraguto (Ferraù) im Kampf getötet worden, wie Boiardo erzählt; es scheint nicht, dass der König noch weitere Kinder hat.

31 OG I 11, S. 34.

32 Vgl. Angelicas Erklärung (OG III 10): „Jo sprezzai mille Eroi, | Mà da la vista di guerrier piagato | Nel curar le sue piaghe | Restai ferita, e ne diuenni amante“.

33 Vgl. OF XIX, 20.

34 Orlando $[=\mathrm{BO}]$. Dramma per musica, Da Rappresentarsi nel Teatro di Sant'Angelo L'autunno dell'anno 1727. In Venezia, 1727, Appresso Martino Rossetti in Merceria all'Insegna della Pace. Das Buch wurde erstmals 1713 (von Giovanni Alberti Ristori) vertont, ein erster Orlando Vivaldis wurde 1714 aufgeführt. Vgl. auch Albert Gier: Orlando geloso. Liebe und Eifersucht bei Ariost und in Grazio Bracciolis Libretto. In: Klaus Hortschansky (Hg.): Opernheld und Opernbeldin im 18. Jahrhundert. Aspekte der Librettoforschung. Ein Tagungsbericht (Schriften zur Musikwissenschaft aus Münster; 1). Hamburg und Eisenach 1991, 5 -70, hier 69-70. 
keiten liegen auf anderen Gebieten: „Schönheit und Liebe“ sind seine Waffen, ein Schwert kann seine „weiche Hand“ nicht führen. ${ }^{35}$

Bei Braccioli wie auch in anderen Libretti entspricht Medoro dem Klischee des verweichlichten Orientalen, und wird so gleichsam zum Antitypus Orlandos. Natürlich repräsentiert jener unkriegerische Medoro nicht die muslimische Streitmacht (und erst recht nicht das muslimische Rittertum, dem er gar nicht angehört): Sacripante, Mandricardo, Ferraù oder der schreckliche Rodomonte liefern hinreichende Beweise ihrer Tapferkeit. ${ }^{36}$ Dennoch scheint der Gegensatz zwischen dem christlichen Helden und dem effeminierten Muslim in den Libretti signifikant.

Nach seinem Orlando schrieb Braccioli das Buch zu Rodomonte sdegnato, das Michelangelo Gasparini in Musik setzte (1714). ${ }^{37}$ Die Geschichte spielt auf der „Insel der Mörderweiber“" 38 in einem Amazonenstaat, der auch bei Ariost, allerdings in gänzlich anderem Zusammenhang, vorkommt. ${ }^{39}$ Nach der Niederlage gegen Karl den Großen vor Paris hat sich Bracciolis Agramante mit seinen Getreuen auf diese Insel geflüchtet. Da sich die Damen wenig gastfreundlich zeig-

35 BO III 9: „Disdicono, mio Sposo | Alla molle tua destra | E al tenero tuo sen | Spada, e furore! | Son bellezza ed amore | L'armi tue, il tuo vigor“.

36 In Nunziato Portas dramma eroicomico Orlando Paladino, das unter anderem von Joseph Haydn vertont wurde (1782; Text mit moderner deutscher Übersetzung: Joseph Haydn: Orlando paladino. Dramma eroicomico in tre atti. Ritter Roland. Heroisch-komische Oper in drei Akten, übers. von Roberto Scoccimarro. In: Innsbrucker Festwochen der Alten Musik $\mathrm{GmbH}$ [Hg.]: Joseph Haydn. Orlando Paladino. Innsbruck 2009, 108-243, http://www.alte musik.at/fileadmin/user_upload/02_Programm/Programmbuch_als_Download/Orlando_ ALM09.pdf, 29. März 2016), treten der hasenherzige Medoro und der gewalttätige, prahlerische Rodomonte gemeinsam auf. Während dieser sich seiner Heldentaten rühmt (vgl. Arie Nr. 6: „Sono il re di Barbaria, | e il valor dell'alma mia | S'ode ovunque rimbombar. | Mostri orribili e giganti | Fatti ho a pezzi come offelle | più che in ciel non vi son stelle $\mid \mathrm{O}$ vi sono arene in mar"), verfällt Medoro in Schockstarre, sobald er von Orlandos Ankunft auf Alcinas Insel erfährt: Angelica beschwört ihn zu fliehen und sich zu verstecken, er aber kann sich nicht entschließen (Arie Nr. 13: „Parto. Ma, oh dio, non posso. | Resto. No, vil mi rendo."). Etwas später scheint er entschlossen, die Auseinandersetzung mit Orlando zu suchen, lässt sich aber von Angelica umstimmen. Zu Beginn des II. Akts hat er an einem menschenleeren Strand Zuflucht gesucht, aber der „einsame schreckliche Ort“ („In questo solitario orrido luogo" [II 3, S. 169]) macht ihm auch wieder Angst.

37 Rodomonte sdegnato [=RS]. Dramma per Musica. Da Rappresentarsi nel Teatro di S. Angelo. Il Carnovale dell'Anno 1714. Del Dottor Grazio Braccioli [...] In Venezia, M.DCCXIV. Appresso Marino Rossetti. In Merzaria all'Insegna della Pace. - Ein Zweitdruck (Titelblatt identisch, bis auf den Zusatz „Seconda Impressione, con nuova Aggiunta") aus dem gleichen Jahr ist bis Szene II 16 mit der Erstfassung identisch; in der Schlussszene des II. Akts (II 17) beginnt Belisa nicht zu delirieren, wenn sie sich auf Alerias Thron setzt (wie im Erstdruck), sondern der Thron verwandelt sich in einen Strudel, der sie verschlingt. - Im III. Akt des Zweitdrucks treffen die Ritter auf eine Hirtengesellschaft, die mit Rundgesang und Pfänderspielen eine Hochzeit feiert. Nachdem Rodomonte Aleria überwunden hat, verliebt er sich in sie, beide werden ein Paar (III 7); Doralice entscheidet sich nicht selbst für Mandricardo, sondern sie bittet Amor um ein Orakel, das ihr jede Hoffnung auf Elbanios Liebe nimmt.

38 Vgl. Argomento, S. 7: „il Regno delle Donne Omicide“; vgl. OF XIX, 57, V. 2.

39 OF XIX, 43-XX, 96: Ein Sturm verschlägt Marfisa, Astolfo und mehrere andere Ritter auf die Insel, wo die Frauen Waffen tragen, während die Männer in Frauenkleidern gehen und Frauenarbeit verrichten; die Ritter können fliehen, nur Astolfo bleibt auf der Insel zurück. 
ten, hat er ihre Hauptstadt belagert, zweifelt aber unterdessen am Erfolg des Unternehmens und ist zum Rückzug entschlossen. Die handelnden Figuren gehören fast ausschließlich Agramantes Heer oder Gefolge an. Die Mörderweiber sind nur durch ihre Königin Aleria vertreten, christliche Ritter sind gar nicht beteiligt.

Die Geschichte liest sich wie eine Illustration der Maxime des Grafen BussyRabutin: "Quand on n'a pas ce que l'on aime, il faut aimer ce que l'on a“:40 Rodomonte liebt Doralice, macht aber zuletz ${ }^{41}$ seiner Gegenspielerin Aleria einen Heiratsantrag. Diese wiederum fühlt sich gleich zu zwei Sarazenenrittern hingezogen, zu Armindo und zum hübschen Elbanio, der auch Doralice gefällt, aber leider - welche Verschwendung - nicht an der Liebe, sondern nur an Waffentaten interessiert ist, weshalb Doralice zuletzt Mandricardo nehmen muss. Einzig das Paar Armindo und Belisa steht von Anfang bis Ende treu zueinander.

Die von Rodomonte und Mandricardo umworbene Doralice erweist sich als oberflächlich und berechnend: Anfangs scheint sie geneigt, Mandricardo zu erhören, aber nur mangels besserer Alternativen. ${ }^{42}$ Wenn der hübsche Elbanio auftaucht, ist sie tief beeindruckt. ${ }^{43}$ Später erklärt sie ihren beiden Langzeit-Verehrern, sie dürften sich nicht wundern, wenn sie Elbanio vorzöge, schließlich sei er der attraktivste von ihnen dreien. ${ }^{44}$ Dennoch erwartet sie, dass Mandricardo und Rodomonte ihrem Idol beistehen (um Freiheit für sich und seine Gefährten zu erringen, will Elbanio es mit zehn Gegnern ${ }^{45}$ aufnehmen). Wer ihr diesen kleinen Gefallen tut, darf immerhin auf einen zärtlichen Blick und eine Liebkosung rechnen. Solche Koketterie passt weder zu den von Montesquieu beschriebenen Haremsdamen noch zu den Frauen in den Geschichten aus Tausendundeine Nacht, die Antoine Galland zwischen 1704 und 1717 in französischer Übersetzung publizierte. ${ }^{46}$ Ähnliches könnte man eher bei zeitgenössischen Pariser salonnières finden.

Rodomonte ist nicht ohne Grund stolz auf seine Körperkraft und Geschicklichkeit im Umgang mit den Waffen (zu Beginn ist er ganz allein in die belagerte Stadt eingedrungen und hat dort offenbar viel Schaden angerichtet) ${ }^{47}$; sein finsterer

40 In einem Brief an Mme de Sévigné vom 23. Mai 1667, in: Madame de Sévigné: Correspondance I (mars 1646 - juillet 1675), hg. von Roger Duchêne (Bibliothèque de la Pléiade). Paris 1972, 86. Der Herausgeber weist (928) den Prolog der Komödie L'Inconnu von Thomas Corneille (1675) als Quelle nach.

41 Nur im Zweitdruck des Librettos (Anm. 37).

42 „In penuria d'amanti“ (RS I 2).

43 „Oh questo è un volto | Che sollecita l'Alma“ (RS I 4).

44 „Non vi stupite; egli è più bel di voi“ (RS II 11).

45 Bei Ariost werden Fremde, die auf die Amazoneninsel kommen, gefangen genommen, es sei denn, einer von ihnen vermöchte zehn Männer im Kampf zu besiegen und im Bett zehn Mädchen zu befriedigen (die er anschließend heiraten muss, denn auf der Insel teilen sich jeweils zehn Frauen einen Mann). Bei Braccioli sind es zehn Statuen, die Aleria durch einen Zauber zum Leben erweckt.

46 Vgl. Heinz Grotzfeld: Antoine Galland. In: Rolf Wilhelm Brednich (Hg.): Enzyklopädie des Märchens. Handwörterbuch der historischen und vergleichenden Erzäblforschung, Bd 5. Berlin und New York 1987, 660-662.

47 RS I 3. 
Blick, ${ }^{48}$ seine grobe Art zu reden ${ }^{49}$ sind aber nicht dazu angetan, eine Frau für ihn einzunehmen. Er ist genügend von sich überzeugt um zuzustimmen, wenn Agramante vorschlägt, Doralice selbst solle ihren Gatten wählen..$^{50} \mathrm{Da}$ sie sich für Mandricardo entscheidet, spuckt er allerdings Gift und Galle und fordert ihn zum Schwertkampf, was Agramante nicht zulässt. ${ }^{51}$ Später, wenn sich zeigt, dass beide Rivalen gegen Elbanio keine Chance haben, verdammt Rodomonte die Frauen in Bausch und Bogen und will gar an Alerias Amazonen Rache nehmen, ${ }^{52}$ die für Doralices Flatterhaftigkeit nun wirklich nichts können.

Rodomonte steht in der Tradition der hünenhaften, über gewaltige Körperkraft verfügenden ,heidnischen' Kämpfer der mittelalterlichen Heldenepik, vom Fierabras der altfranzösischen Chanson de geste (12. Jahrhundert) über Rainouart ${ }^{53}$ in der Wilhelmsgeste bis zu Luigi Pulcis Morgante (1478). Durch sein stark ausgeprägtes Selbstbewusstsein macht er sich nicht lächerlich, da er seinen Worten Taten folgen lässt; komisch ist (bei Ariost) allenfalls seine erfolglose Werbung um Doralice, und (in Bracciolis Libretto) die wütende Reaktion darauf. Obwohl die Figur kaum spezifisch muslimische Züge aufweist, scheint es bezeichnend, dass christliche Ausprägungen des durch Rodomonte verkörperten Typus in der mittelalterlichen und frühneuzeitlichen Tradition zu fehlen scheinen.

Während Ariost im Wesentlichen die kriegerischen, vor allem aber erotischen Abenteuer einer kultur- und religionsübergreifenden Oberschicht erzählt, wobei die Überlegenheit der christlichen gegenüber der muslimischen Religion als selbstverständlich vorausgesetzt, aber nur an wenigen Stellen thematisiert wird, stehen sich bei Tasso die Christen als Gottesstreiter und ihre muslimischen Gegner als Armee der Hölle gegenüber. Beim Teufelskonzil, das im vierten Canto (GL IV, 1-19) der Gerusalemme liberata geschildert wird, ${ }^{54}$ fordert Plutone die Höllengeister auf, den Erfolg des Kreuzzugs mit allen Mitteln zu verhindern; gleich darauf wird erzählt, wie Idraote, der Fürst von Damaskus, seine Nichte Armida ins christliche Lager schickt, um dort Unruhe zu stiften, ${ }^{55}$ zweifellos hat ihn ein Dämon auf diese Idee gebracht.

48 Vgl. Doralice: „Il fosco ciglio, e la sdegnosa voce | In donzella, Signore, | Son di spavento, e non cagion d'amore" (RS I 12).

49 Doralice: „Che rozi accenti“ (RS II 2).

50 RS II 2.

51 RS II 3.

52 „Donne a regnar, donne dell'vom nimiche | Non soffre lo sdegnato Rodomonte“ (RS II 12).

53 Rainouart ist in Frankreich aufgewachsen (als Küchenjunge im Palast Ludwigs des Frommen), erweist sich aber (in der Chanson de geste Aliscans, zweite Hälfte des 12. Jahrhunderts) zuletzt als Sohn des Sarazenenkönigs.

54 Torquato Tasso: Gerusalemme liberata $[=\mathrm{GL}]$, hg. von Lanfranco Caretti (I Meridiani Collezione). Mailand 2006.

55 GL IV, 20-26. 
Muslime, die Tasso positiv zeichnet, haben stets irgendeine Affinität zum Christentum: Die Heldenjungfrau Clorinda ist Tochter christlicher Eltern, ${ }^{56}$ die Sarazenenprinzessin Erminia liebt heimlich den christlichen Ritter Tancredi und entfernt sich deshalb innerlich von der Religion ihrer Väter. Selbst die teuflische Armida kann um den Preis ihrer Konversion ${ }^{57}$ rehabilitiert werden.

Bis etwa zur Mitte des 18. Jahrhunderts orientierten sich Librettisten, die den Armida-Stoff behandeln, gewöhnlich an Tassos manichäistischem Religionsverständnis. ${ }^{58}$ Besonders deutlich wird das in Giacomo Rossis (und Aaron Hills) Rinaldo-Libretto für Georg Friedrich Händel ${ }^{59}$ (London 1711): Der christlichen Sphäre sind hier eine arkadische Natur und das Licht des Tages, Armida Monstren, Dämonen und das Dunkel der Nacht zugeordnet. Bei ihrem ersten Auftritt ${ }^{60}$ erscheint die Zauberin auf einem von feuerspeienden Drachen gezogenen Wagen, als Eskorte wünscht sie sich „schreckliche Furien“; Rinaldos Verlobte Almirena treffen wir unmittelbar danach an einem „Lustort mit Quellen, Alleen und einer Voliere, in der die Vögel fliegen und singen“. ${ }^{61}$ Armida entführt sie mittels einer „schwarzen Wolke voll schrecklicher Monstren, die unter lautem Gebrüll Feuer und Rauch speien“;62 Almirena klagt später, sie sei „mit der Kraft des Höllenschlunds aus dem teuren Himmel ihres Glücks“ gerissen worden. ${ }^{63}$ Wenn Armida die Gestalt Almirenas annimmt, um Rinaldo zu verführen, erkennt er in ihr „die Hölle in der Maske des Paradieses".64 Auch die Normsysteme und Verhaltensmuster der Christen sind denen der Muslime diametral entgegengesetzt: Zu Beginn scheint das Ziel, die Eroberung Jerusalems, zum Greifen nahe, und Rinaldo würde gern gleich Hochzeit

56 Sie ist die Tochter des äthiopischen Königspaars, vgl. GL XII, 21-25.

57 Zuletzt gibt sie sich ganz in die Hand Rinaldos (GL XX, 136, V. 7/8: „- Ecco l'ancilla tua; d'essa a tuo senno | dispon, - gli disse - e le fia legge il cenno."), der sie natürlich auffordern wird, sich taufen zu lassen.

58 In den Armida-Opern der zweiten Hälfte des 18. und des frühen 19. Jahrhunderts wird der Gegensatz von Himmel und Hölle zum Konflikt zwischen (aus der Rolle der Protagonisten in der Gesellschaft resultierender) Pflicht und individuellen Gefühlen; dazu Albert Gier: Ecco l'ancilla tua... Armida in der Oper zwischen Gluck und Rossini (mit einem Seitenblick auf Antonín Dvořák). In: Achim Aurnhammer (Hg.): Torquato Tasso in Deutschland. Seine Wirkung in Literatur, Kunst und Musik seit der Mitte des 18. Jabrhunderts. Berlin und New York 1995, 643660. Zur Stoffgeschichte vgl. auch Jérôme Pesqué: Quatre siècles de livrets d'opéra en compagnie de Renaud et d'Armide. In: Danielle Buschinger (Hg.): Quatre siècles de livret d'opéra. Actes du Colloque de Saint-Riquier (9-10 et 11 Octobre 2002). Amiens 2004, 170-184.

59 Vgl. auch Klaus Pietschmann: Rinaldo (HWV 7a / b). In: Arnold Jacobshagen und Panja Mücke (Hg.): Händels Opern (Das Händel-Handbuch; 2), Teilbd. 2. Laaber 2009, 54-62. Rinaldo $[=\mathrm{RR}]$. Opera. Da rappresentarsi nel Reggio Teatro a Londra. Printed by Tho. Howlatt [...] London MDCCXI. In: E. T. Harris (Hg.): The Librettos of Handel's Operas, Bd. 2. New York und London 1989, 1-75, hier I 5.

61 RR I 6, S. 24: „Luogo di Delizie con Fonti, Viali, ed Vccelliere, in cui Volano, e cantano gli Vccelli."

62 RR I 7, S. 28: „discende una Nube negra ripiena di Mostri horribili, che mandano fuori Fiamme, e fumo, con gran Muggiti“.

63 RR II 4, S. 38: „Colla Forza d'Abisso | Rapirmi al caro Ciel de’ miei Contenti!“

64 RR II 7, S. 36: „V' è l'Inferno con un vel di Paradiso.“ 
machen, aber Goffredo verfügt: Erst die Arbeit, dann das Vergnügen, und Almirena stimmt zu. ${ }^{65}$ Dagegen fordern die Sirenen als Sprachrohr der Sphäre Armidas die Liebenden auf, „den Mai ihrer jungen Jahre“ der Liebe zu weihen und sich nicht durch „ein Trugbild der Ehre“ davon abhalten zu lassen. ${ }^{66}$ Während Almirena und Rinaldo einander selbstverständlich treu sind, würde Armidas Liebhaber Argante, der König von Jerusalem, die Zauberin nur allzu gern mit der hübschen Christin betrügen, ${ }^{67}$ obwohl er vor der Eifersucht und „höllischen Wut" seiner Freundin einen Heidenrespekt - c'est le cas de le dire - hat; ${ }^{68}$ dabei hätte Armida gar keinen Grund sich aufzuregen, ist sie doch selbst von dem hübschen Rinaldo fasziniert.

In Libretti der romantischen Zeit finden sich solche Symmetrien nicht mehr. Viele Texte handeln von christlich-muslimischen Familiengeschichten, und nicht immer sind die Christen die verständnisvolleren und barmherzigeren. Das gilt schon für Gaetano Rossis Buch zu Giacomo Meyerbeers Crociato in Egitto ${ }^{69}$ (Venedig 1824): Eine Truppe des Johanniterordens wurde während des sechsten Kreuzzugs (12481254) in Ägypten fast vollständig aufgerieben; Armando, der einzige Überlebende, verwandelt sich in einen Ägypter, tritt unter dem Namen Elmireno in den Dienst des Sultans von Damiette und macht in den folgenden Jahren eine beachtliche militärische Karriere. Sein Onkel Adriano, der Großmeister des Ordens, und seine Verlobte Felicia halten ihn für tot. Armando verliebt sich in Palmide, die Tochter des Sultans (er erregt ihre Aufmerksamkeit, indem er „unter den Mauern ihres abgelegenen Harems ${ }^{670}$ eine Romanze singt). Seinetwegen wird sie Christin, die beiden heiraten heimlich und haben einen Sohn, der, wenn die Handlung der Oper einsetzt, vielleicht vier oder fünf Jahre alt ist.

Sultan Aladino schätzt ,Elmireno' sehr und will ihm seine Tochter zur Frau geben, ${ }^{71}$ ohne zu ahnen, dass die beiden - nach christlichem Ritus - längst verheiratet sind. Die Situation eskaliert, sobald Adriano zu Friedensverhandlungen nach Damiette kommt: Er erkennt Armando, schimpft ihn einen Verräter und will ihm nur verzeihen, wenn sein Neffe dem Sultan seine wahre Identität offenbart, zum Orden zurückkehrt und Felicia heiratet (obwohl diese mit Rücksicht auf Palmide

65

66 RR II 3, S. 34: „Il vostro Maggio | De’ bei verdi Anni | O cari Amanti | Sempre costanti | Sfiorate in Amor; | Nè un falso Raggio | D’Honor v'affanni, | Ch' e sol beato | Chi Amante amato | Possede un bel Cor."

67

68

69 Teatro La fenice. Venedig 1824, http://www.librettidopera.it/crociato/crociato.html, 29. März 2016.

70 CE I 12: „Sotto le mura| Del mio remoto harem“.

71 Bei der Hochzeitszeremonie (die abgebrochen wird, weil Armando im Habit der Johanniter erscheint und sich zu erkennen gibt) wird der Gran Profeta, also Mohammed, als schützende Instanz angerufen. 
und das Kind bereit wäre, auf ihren Verlobten zu verzichten). Aladino ist empört, dass man ihn derart hintergangen hat, und lässt Armando ins Gefängnis werfen. Er befiehlt sogar, das Kind, seinen Enkel, zu töten, ehe er sich von Palmide umstimmen lässt und verzeiht.

Adriano dagegen scheint unversöhnlich. Erst als er von Palmides Konversion erfährt, gibt er nach - unter der Bedingung, dass sie sich öffentlich zum Christentum bekennt und den Johannitern folgt. Auf den Einwand, sie könne ihren Vater nicht verlassen, antwortet er: „Il tuo Dio prima“72, was nun allerdings recht fanatisch klingt. Mit dem Abfall seiner Tochter vom Glauben ihrer Väter kann Aladino sich aber nicht abfinden, eine bewaffnete Auseinandersetzung, die mit der Vernichtung der Christen enden müsste, scheint unvermeidlich.

Um dennoch ein glückliches Ende herbeizuführen, zitiert Rossi den Schluss von Pietro Metastasios dramma per musica Ezio (1728): Aladinos Großwesir Osmino hat eine Verschwörung angezettelt, um den Sultan zu stürzen (wie Metastasios Patrizier Massimo Kaiser Valentiniano beseitigen wollte), Armando rettet Aladino das Leben (wie der von Valentiniano zu Unrecht verdächtigte und eingekerkerte Feldherr Ezio seinem undankbaren Herrn im Augenblick der Gefahr beistand). Natürlich ist der Sultan daraufhin bereit zur Versöhnung, er stimmt der Verbindung von Palmide und Armando nachträglich zu, und mit den Johannitern wird er künftig in Frieden leben.

Unter den hier betrachteten Opern ist Il crociato in Egitto die erste, in der zumindest en passant von einem Harem die Rede ist; auch „der Prophet“ wird einmal erwähnt. ${ }^{73}$ So etwas wie Lokalkolorit ist damit zumindest angedeutet; die Libretti des frühen 18. Jahrhunderts hatten einen Bezug auf muslimische Institutionen und Lebensverhältnisse noch bewusst vermieden. ${ }^{74}$

Knapp zwanzig Jahre nach Giacomo Meyerbeers Crociato lässt Verdis Librettist Temistocle Solera in I Lombardi alla prima crociata ${ }^{75}$ (Mailand 1843) Acciano, den Tyrannen von Antiochia, und seine Gefolgsleute den „allah terribile“ anrufen, er möge vom Himmel herabsteigen, um die Kreuzritter, die als Vergewaltiger, Räuber und Mörder bezeichnet werden, zu bestrafen. ${ }^{76}$ Andererseits beschimpfen die

72 CE II 14.

73 Siehe Anm. 71.

74 Bracciolis Orlando finto pazzo-Buch (für Vivaldi, 1714) rekurriert für den Zauber, den Ersilla (sie entspricht Boiardos Falerina, wie das Personenverzeichnis präzisiert) und Tigrinda ausführen, noch auf antike Vorstellungen: Der I. Akt spielt in einem Pluto und Demogorgon (einem Erdgeist) geweihten Tempel, in dem es Altäre für Hekate und Phlegethon gibt; Ersilla ruft den „hohen Herrn des Avernus“ (Pluto) an, ein Schwert, das bei der Zeremonie Verwendung findet, ist „dem König der Styx“ (Pluto) geweiht, et cetera; vgl. Orlando finto pazzo. Drama per musica da Rappresentarsi in S. Angelo l'Autunno 1714 del Dottor Grazio Braccioli [...] in Venetia per Marino Rossetti in Merceria alla Pace.

75 Temistocle Solera und Giuseppe Verdi: I Lombardi alla prima crociata [=LC], Dramma Lirico. Teatro alla Scala, Mailand 1843, http://www.librettidopera.it/lombacro/lombacro.html, 29. März 2016.

76 LC II 1. 
Christen den „stolto allah“, dem eine „heilige Stimme“ das Ende seiner Tage ankündigt. ${ }^{77}$

Auch I Lombardi sind zunächst und vor allem eine Familiengeschichte: Pagano liebte Viclinda, sie aber hat sich für seinen Bruder Arvino entschieden. Der Verschmähte floh, nachdem er seinen glücklichen Rivalen niedergestochen hatte; Jahre später kehrt er aus dem Exil zurück, um seine Rache zu vollenden. Er will den Bruder töten, bemerkt aber nicht, dass nicht dieser, sondern ihrer beider Vater in Arvinos Zimmer schläft, und ermordet ihn. Um seine Schuld zu sühnen, zieht sich Pagano als Einsiedler nach Palästina zurück und findet in den Akten II bis IV mehrfach Gelegenheit, den Kreuzfahrern aus der Lombardei, die unter Arvinos Kommando stehen, nützlich zu sein, bis er beim Sturm auf die Mauern Jerusalems tödlich verwundet wird.

Der Tyrann Acciano hat nur einen kurzen Auftritt in der ersten Szene des II. Aktes, außer den Schmähreden gegen die Kreuzfahrer darf er kaum etwas singen oder sagen. Seine Frau Sofia ist längst heimlich zum Christentum konvertiert, ihrer beider Sohn ist ebenfalls bereit, diesen Schritt zu tun - vor allem einer christlichen Gefangenen wegen, die er liebt und die seine Gefühle erwidert; ${ }^{78}$ es handelt sich um Arvinos Tochter Giselda, die von Accianos Leuten aus dem Lager der Christen entführt wurde. Arvino, Pagano und ihre Soldaten dringen in Accianos Harem ein, um Giselda zu befreien (hier ist vom Harem nicht nur die Rede, er wird sogar auf der Bühne gezeigt). Als die junge Frau hört, Acciano und Oronte seien beim Angriff auf den Palast ums Leben gekommen, verdammt sie in ihrem Schmerz über den Tod des Geliebten den Kreuzzug als „schändlichen Wahnsinn“, die nur dazu diene, die Gier nach Gold zu befriedigen, und dem Willen Gottes zuwiderlaufe:

$\begin{array}{ll}\text { No! Giusta causa } & \text { non è iddio } \\ \text { La terra spargere } & \text { di sangue umano; } \\ \text { È turpe insania } & \text { non senso pio } \\ \text { Che all'oro destasi } & \text { del monsulmano! } \\ \text { Queste del cielo } & \text { non fur parole... } \\ \text { No, dio no'l vuole } & \text { no, dio no'l vuole! }\end{array}$

Diese Rede mochte noch in den Ohren mancher Zeitgenossen Giuseppe Verdis blasphemisch klingen. Arvino hätte seine Tochter dafür getötet, wenn Pagano ihn nicht zurückgehalten hätte.

Im III. Akt stellt sich heraus, dass Oronte nicht tot, aber lebensgefährlich verletzt ist; Giselda erkennt, dass er nicht überleben wird, und klagt den „grausamen

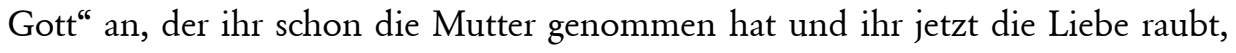

„Nein! Es ist bei Gott keine gerechte Sache, die Erde mit menschlichem Blut zu tränken; es ist schändlicher Wahnsinn, nicht fromme Vernunft, der vom muslimischen Gold geweckt wird! Das waren nicht die Worte des Himmels ... Nein, Gott will es nicht, Gott will es nicht!“ Der letzte Vers negiert expliziert den Schlachtruf der Kreuzritter: „Dieu le veut!“. 
die ihr einziger Tost war. ${ }^{80}$ Pagano tadelt sie für diese blasphemischen Reden. Oronte stirbt, unmittelbar nachdem er aus Paganos Hand die Taufe empfangen hat; den Leib konnte der fromme Mann nicht retten, aber wenigstens für die Seele ist damit gesorgt. Im letzten Akt wird Giselda durch eine Vision, die ihr Oronte unter den seligen Geistern im Himmel zeigt, wunderbar getröstet. ${ }^{81} \mathrm{Ob}$ für den Zuschauer das verheißene Paradies - und die Schlußapotheose, das Bild der Stadt Jerusalem, über der die Fahnen der Kreuzritter wehen - oder Giseldas Anklagen gegen Gott und die Menschen schwerer wiegen, wird wesentlich von seinen eigenen religiösen Überzeugungen abhängen. Das Lombardi-Libretto scheint jedenfalls kaum geeignet, die Vorstellung vom Agnostiker oder Atheisten Verdi zu entkräften. ${ }^{82}$

Auffällig viele Krypto-Christen oder Beinahe-Christen finden sich unter den Muslimen der Kreuzzugsopern; Ruggiero bei Ariost, Clorinda bei Tasso, und in der Zeit der Romantik werden es noch mehr: Armando und Palmide in Meyerbeers Crociato, Sofia und Oronte in Verdis Lombardi. In der Frühen Neuzeit sind Ruggieros Herkunft (aus christlicher Familie) und seine Sympathie für die Religion seiner Ahnen natürlich ein Beweis für die Überlegenheit des Christentums über den Islam, der einen so vortrefflichen Ritter wie Ruggiero anscheinend nicht aus eigener Kraft hervorzubringen vermag. Im frühen 19. Jahrhundert, einer Zeit radikaler Veränderungen in Politik, Gesellschaft, Wirtschaft und Technik, die nicht wenige Menschen überfordert haben dürften, mag das Motiv noch eine andere Bedeutung haben: Der gläubige Christ Armando ist als Muslim ,Elmireno' ebensowenig an seinem rechten Platz wie Verdis Manrico (Il Trovatore), der eigentlich Graf Luna ist, als Räuberhauptmann. Indem solche Plots die Malaise dieser Figuren fokussieren und damit ins Bewusstsein der Zuschauer heben, wird deren eigener Orientierungsverlust nicht kompensiert, aber vielleicht erträglicher gemacht.

Im 19. Jahrhundert, das zeigen Verdis Lombardi sehr deutlich, bietet die Kreuzzugsthematik auch Gelegenheit, zu aktuellen Problemen Stellung zu beziehen und etwa Kritik an religiösem Fanatismus, Kolonialismus oder Imperialismus zu üben. Dafür sei abschließend ein weiteres Zeugnis angeführt: Richard Wagner verfasste zwischen 1841 und 1843 den Libretto-Entwurf Die Sarazenin. ${ }^{83}$ Hauptfigur ist Manfred, der illegitime Sohn Kaiser Friedrichs II., der seine Ansprüche auf die Krone nicht durchzusetzen vermochte und 1266 im Kampf gegen Karl von Anjou den Tod fand. Zu Beginn erinnert die ,Prophetin' Fatima (die Titelheldin) an den

80 LC III 6: „Tu la madre a me togliesti, | M'hai serbata a dì funesti... | Sol conforto è al pianto mio | Questo amore, e il togli a me... | Tu crudel..."

81 LC IV 2.

82 Vgl. auch Albert Gier: Leere Transzendenz in der säkularisierten Welt. Verdi, Requiem, Stiffelio - Wagner, Jesus von Nazareth, Parsifal. In: Norbert Abels (Hg.): Verdi « Wagner. Folkwang Symposion 2013. 14 Beiträge zu ibrem 200. Geburtstag. Frankfurt am Main 2014, 71-91.

83 Richard Wagner: Neue Text-Ausgabe chronologisch und vollständig, hg. von Rüdiger Jacobs, Bd III. Halle und Frankfurt am Main 2013, 157-190 [=WS]. 
fünften Kreuzzug (1228-1229):84 „Der Templer niedre Rotte“ habe den Kaiser verraten und dem Sultan al-Kamil (ca. 1180-1238) ausliefern wollen, aber Zelima, „der Gläubigen Schönste“, habe diesen dazu bewegen können, das Angebot nicht anzunehmen und Friedrich zu warnen. Die beiden Herrscher „schwuren ew'ge Freundschaft sich" (der historische Friedrich schloß mit al-Kamil einen Vertrag, der die Rückgabe von Jerusalem, Bethlehem und Nazareth an die Christen regelte), Zelima wurde Friedrichs Geliebte (wie sich am Ende herausstellt, ist Fatima ihrer beider Tochter). Wagner lässt die Utopie einer friedlichen Koexistenz der Religionen aufscheinen:

Beglückt umarmte sich Christ und Muselmann. Denn er, der große Kaiser, war nicht Christ, nicht Muselmann, er war ein Gott, und als ein Gott verehrt lebt er noch heut im Morgenland. (WS, 159)

Musiktheater-Werke, die historische oder fiktionale Episoden aus der Chronik der Kreuzzüge zum Vorwurf nehmen, können entweder die Gemeinsamkeiten muslimischer und christlicher Ritter (so in den Opern nach Ariost) oder ihre radikale Verschiedenheit im Sinne eines Gegensatzes von Gut und Böse, Gott und Teufel oder Ähnlichem (in den Opern nach Tasso) behaupten; sie können einen nahezu zeit- und ortlosen Orient in Szene setzen wie im Ancien Régime oder Lokal- und Zeitkolorit zumindest andeuten wie in romantischer Zeit. Zudem können sie Bezüge zur Gegenwart herstellen, indem sie ihre Figuren (ironisch persiflierend wie in den Ariost-Opern) als Zeitgenossen porträtieren oder indem sie (wie bei Wagner und Verdi) eine für die eigene Zeit bedeutsame, allgemeine Aussage formulieren. Dem bunten Strauß von Möglichkeiten entspricht die Vielfalt musikalischdramatischer Erscheinungsformen, von denen im Rahmen dieses Beitrags nur wenige Beispiele präsentiert werden konnten. ${ }^{85}$

84 WS, 158-159.

85 Vgl. auch Albert Gier: „,Oh Richard ô mon roi‘. Richard Löwenherz im Musiktheater“. Erscheint in den Akten der Ringvorlesung „, Richard Löwenherz - ein europäischer Herrscher im Zeitalter der Konfrontation von Christentum und Islam“, Universität Bamberg, Zentrum für Mittelalterstudien, Sommersemester 2016. 
